\title{
On the Conservation of Information in Quantum Physics
}

\author{
Marco Roncaglia* \\ Physics Department and Research Center OPTIMAS, University of Kaiserslautern, Germany
}

(Dated: September 11, 2017)

\begin{abstract}
According to quantum mechanics, the informational content of isolated systems does not change in time. However, subadditivity of entropy seems to describe an excess of information when we look at single parts of a composite systems and their correlations. Moreover, the balance between the entropic contributions coming from the various parts is not conserved under unitary transformations. Reasoning on the basic concept of quantum mechanics, we find that in such a picture an important term has been overlooked: the intrinsic quantum information encoded in the coherence of pure states. To fill this gap we are led to define a quantity, that we call coherent entropy, which is necessary to account for the "missing" information and for re-establishing its conservation. Interestingly, the coherent entropy is found to be equal to the information conveyed in the future by quantum states. The perspective outlined in this paper may be of some inspiration in several fields, from foundations of quantum mechanics to black-hole physics.

PACS numbers: 03.67.-a, 03.65.Ta, 04.70.Dy
\end{abstract}

Introduction Every physicist is confident with the principle of energy conservation and aware on its importance and implications. During time evolution in isolated systems, energy is converted from one form to another or transferred between different subsystems, provided the total amount remains the same. However, when we consider information the picture is not so clear. In quantum physics, the conservation of information has been related to no-cloning theorems [1], but apparently it has not been associated to a suitable conserved quantity. It is a well-known fact that the von-Neumann entropy $S(\rho)=-\operatorname{Tr}\left(\rho \log _{2} \rho\right)$ of any isolated quantum system with density operator $\rho$ does not change in time. This is a consequence of the fact that $S(\rho)$ depends only on the spectrum of $\rho$, and the unitarity of time evolution preserves the spectrum at the quantum level. In this sense, people say that any physical process governed by quantum mechanics information is never lost. However, this is a static vision that involves isolated quantum systems. So far, there is no complete theory able to clearly describe how quantum information "flows" between interacting systems, accounting for a correct balance at any time.

Whenever a system $A$, which is initially in a pure state $\rho_{A}=\left|\psi_{A}\right\rangle\left\langle\psi_{A}\right|$, is no more isolated because it interacts with another system $B$, we start to observe the increase of its mixedness, quantified by the entropy $S\left(\rho_{A}\right)$, where $\rho_{A}=\operatorname{Tr}_{B}\left(\rho_{A B}\right)$. If we look at the total entropy $S\left(\rho_{A}\right)+S\left(\rho_{B}\right)$, we see that it can only increase with time as a consequence of the subadditivity property $S\left(\rho_{A B}\right) \leq S\left(\rho_{A}\right)+S\left(\rho_{B}\right)$ [2, 3]. Moreover, interaction creates some correlations between $A$ and $B$, with an additional contribution of information, which can be measured by their mutual information $I_{A: B}$. Such a description is not governed by a balance equation of a conserved quantity, giving the illusion that information is created via interaction. Where does all this information come from? It is clear that entropy is not the right quantity to describe the full informational content of quantum states.

In this paper, we propose to treat separately the coherent and the incoherent contributions of the informational content of quantum states. Starting from the very basic principles of quantum mechanics, we will introduce the concept of coherent entropy, a quantity able to detect the information that quantum states convey in time. In this context, pure states contain more coherent information than mixed states, as the missing information has been converted into correlations with the environment. We will find that the coherent information, associated to genuinely quantum phenomena, is indeed conserved under unitary processes.

Informational content of quantum states A pure state is the eigenstate of some complete observable, whose measurement gives a fully predictable outcome. Hence, the associated zero entropy accounts for the absence of information obtainable by a repeated measurement over many copies. However, it appears reductive to attribute a zero informational content to pure states. In fact, in contrast with single deterministic classical states, they represent the ideal resource for performing quantum tasks, like interference phenomena, quantum computation, and so on. An indicator beyond the (von-Neumann) entropy is needed to describe such information.

Let us take the textbook example of one qubit, i.e. a pure state in dimension $d=2$ of the Hilbert space. Observables are represented by the set of Pauli matrices with eigenvalues $\{+1,-1\}$, where $\sigma^{z}$ has eigenstates $\{|\uparrow\rangle,|\downarrow\rangle\}$, and $\sigma^{x}$ has eigenstates $\{|+\rangle,|-\rangle\}$. If our source emits quantum objects in the state $|\uparrow\rangle=(|+\rangle+|-\rangle) / \sqrt{2}$, a measurement along $\sigma^{x}$ gives a random sequence of values +1 and -1 , with equal probability $p_{+}=p_{-}=1 / 2$. In this case the entropy of information is one bit, i.e. the maximum obtainable for a dichotomic variable. Differently, if the observer measures along $\sigma^{z}$, he obtains the constant sequence of +1 , with zero entropy. This property of detecting different entropies under different mea- 


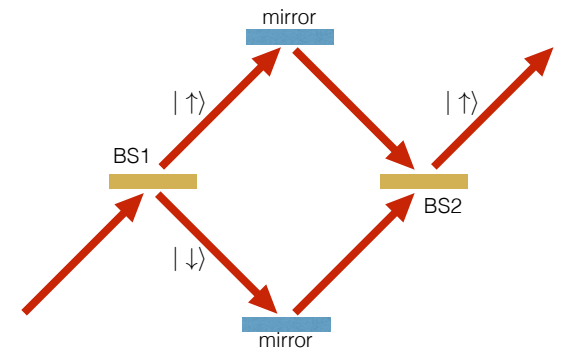

Figure 1. In a Mach-Zehnder interferometer single photons after the beam splitter BS1 produce a signal with maximum entropy $S=1$. In fact, a photon detection just after BS1 gives random sequences of +1 and -1 (photons in the upper and the lower branch, respectively), with equal probability. Instead after BS2, the photons are all found in the $|\uparrow\rangle$ state, which yield a signal with zero entropy. Such a recombination into a single state is a genuinely quantum phenomenon, since it is due to interference between the two coherent beams coming from BS1.

surements is genuinely quantum, as it is ultimately due to interference: in the present example, distinct states $(|+\rangle,|-\rangle)$ coherently recombine into a single state $(|\uparrow\rangle)$, thanks to their well-defined relative phases.

In quantum optics, this example is realized by the Mach-Zehnder interferometer (see Fig[1), where the path of a single-photon beam is split in two different directions by a $50 \%$ reflective mirror (the beam splitter BS1) and then constructively recombined into a single path by a second beam splitter BS2. A measure of the presence of the photon in the upper $(|\uparrow\rangle)$ or lower branch $(|\downarrow\rangle)$ after BS1 gives a random sequence of +1 and -1 , with equal probability. At the output after BS2, the photon detection produces a steady sequence of +1 's, which yield a signal with zero entropy. As the role of the beam splitter is to rotate the basis of measurement, we deduce that the entropy of the detected signal depends in essence on the observable we choose.

In the case of mixed states the effect of interference is reduced, so in every measurement basis we expect to have a residual randomness with a nonvanishing entropy; the limit case is the completely mixed state, where the entropy of the outcome is maximal for every measurement. Notice that an observer who detects the signal after BS1 is not able to distinguish between the pure case and the totally mixed one as they have the same statistics. However, it is important to introduce a measure able to account for the information carried the in the former coherent case, different from the entropy $S(\rho)$ which only quantifies the incoherent information in the latter case.

Coherent entropy Once the measurement basis is fixed, the probability of obtaining a given output is encoded in the diagonal elements $\rho_{i i}, i=1, \ldots, d$ of the density operator in that basis. The entropy of the output measurements is given by the diagonal entropy of $\rho$, which we define as $S(\tilde{\rho})$, where $\tilde{\rho}_{i j}=\delta_{i j} \rho_{i j}$ is the density operator where all the off-diagonal entries have been set to zero. Now, we define the coherent entropy as

$$
S_{c}(\rho)=\max _{\sigma \in \mathcal{U}_{\rho}}[S(\tilde{\sigma})]-\min _{\sigma \in \mathcal{U}_{\rho}}[S(\tilde{\sigma})],
$$

where $\mathcal{U}_{\rho}=\left\{U \rho U^{\dagger}: U \in \mathcal{M}_{d \times d}, U U^{\dagger}=\mathbb{I}\right\}$ is the set of all matrices which are unitarily equivalent to $\rho$. In other words, $S_{c}(\rho)$ measures the difference between the maximal and the minimal entropy of the outputs obtained by measuring $\rho$ over any possible observable. As we have seen, this difference accounts for all the interference effects, so it has to be intended as a measure [4] of the coherent informational content of $\rho$. As it should be for a proper intrinsic property of a quantum state, $S_{c}(\rho)$ is independent of any choice of measurement made by the experimenter, i.e. it is invariant under local unitary transformations. The apparently hard optimization problem of evaluating Eq.(1) eventually leads to a very simple result:

$$
S_{c}(\rho)=\log _{2} d-S(\rho) .
$$

Proof - For every density operator $\sigma \in \mathcal{U}_{\rho}$, we have $S(\tilde{\sigma})=-\operatorname{Tr}(\tilde{\sigma} \log \tilde{\sigma})=-\operatorname{Tr}(\sigma \log \tilde{\sigma})$, as $\tilde{\sigma}$ is the diagonal part of $\sigma$. The difference

$$
S(\tilde{\sigma})-S(\sigma)=\operatorname{Tr}[\sigma(\log \sigma-\log \tilde{\sigma})]=S(\tilde{\sigma} \| \sigma) \geq 0
$$

due to the non negativity of the relative entropy $S(\tilde{\sigma} \| \sigma)$, and the equality holds if $\tilde{\sigma}=\sigma[5]$. Since $S(\sigma)=S(\rho)$, $\forall \sigma \in \mathcal{U}_{\rho}$, we get $\min _{\sigma \in \mathcal{U}_{\rho}}[S(\tilde{\sigma})]=S(\rho)$. Intuitively, the operation of deleting the off-diagonal elements is a decoherence operation (i.e. entropy increasing), which has no effect only in the basis where $\rho$ is already diagonal. Regarding the first term in Eq. (1), we can say it is equal to the entropy of the totally mixed state, namely $\log _{2} d$. Indeed, it is a less well-known fact that under the most general unitary group, every density operator can be transformed into the matrix with diagonal elements uniformly equal to $1 / d[8]$.

The assignment of a purely quantum entropic measure $S_{c}(\rho)$ to a state through Eq. (2) says that the informational content of a pure state is all coherent, while its (von-Neumann) entropy is zero. On the opposite side, in a totally mixed state the information is entirely incoherent. Notice that the sum of $S_{c}(\rho)$ and $S(\rho)$ is always equal to $\log _{2} d$ for every state, meaning that every quantum state (at variance with classical ones) produces a constant unavoidable maximal randomness in the outcomes.

Though the expression in the r.h.s. of (2) has already appeared in the literature [6] as the amount of thermodynamic work that $\rho$ can extract from a heat bath or the number of pure state distillable from $\rho$ [7], it was not obtained and interpreted in the present way. In the following section, we provide another striking interpretation of the same formula. 


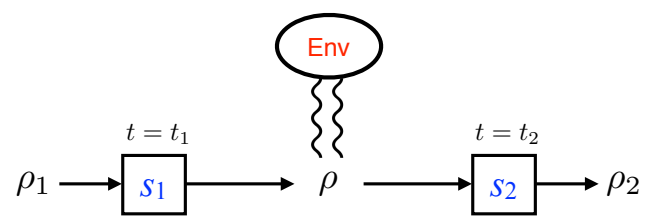

Figure 2. Scheme of measurements during time. The state $\rho$ conveys information between past $s_{1}$ and future $s_{2}$ measurement outputs. Some information has "leaked" into the environment during decoherence, but the total information is conserved.

Time correlations In this section we want to show that the coherent entropy of a given a state $\rho$ expressed in Eq. 2. is exactly equal to the amount of information conveyed between past and future measurements, due to quantum self-correlations in time. We consider the scheme depicted in Fig 2 the state of interest $\rho$ is prepared by the measurement of some observable on $\rho_{1}$ at time $t_{1}$ and a subsequent decoherence through interaction with the environment. At a later time $t_{2}$, the quantum state undergoes another measurement. The time correlation between the two measurement signals $s_{1}, s_{2}$, with probabilities $p\left(s_{1}\right)$ and $p\left(s_{2}\right)$, is estimated by their mutual information

$$
I_{1: 2}=\sum_{s_{1}, s_{2}} p\left(s_{1}, s_{2}\right) \log _{2}\left(\frac{p\left(s_{1}, s_{2}\right)}{p\left(s_{1}\right) p\left(s_{2}\right)}\right)
$$

where $p\left(s_{1}, s_{2}\right)$ is the joint probability. The quantum state can be viewed as a channel, whose capacity is obtained by maximizing $I_{1: 2}$ over all inputs.

For the sake of clarity, we present here a detailed calculation of $I_{1: 2}$ in the case of one qubit and a depolarizing channel as a model of decoherence. Assume that initially we have the state $\rho_{1}=\frac{1}{2}\left(\mathbb{I}_{2}+\mathbf{r}_{1} \cdot \boldsymbol{\sigma}\right)$ represented by the vector $\mathbf{r}_{1}$ inside the Bloch sphere. At time $t_{1}$, we decide to perform a projective measurement along the direction described by the unit vector $\hat{\mathbf{n}}_{1}$. The outcome $s_{1}= \pm 1$ will correspond the state $P_{\hat{\mathbf{n}}_{1}}^{s_{1}}=\frac{1}{2}\left(\mathbb{I}_{2}+s_{1} \hat{\mathbf{n}}_{1} \cdot \boldsymbol{\sigma}\right)$ with probability $\operatorname{Tr}\left(\rho_{1} P_{\hat{\mathbf{n}}_{1}}^{s_{1}}\right)=\frac{1}{2}\left(1+s_{1} \mathbf{r}_{1} \cdot \hat{\mathbf{n}}_{1}\right)$. The subsequent depolarizing channel will simply reduce the length of the Bloch vector $\hat{\mathbf{n}}_{1} \rightarrow \mathbf{n}_{1}$, without changing its direction. Finally, at time $t_{2}$, we perform a second projective measurement along $\hat{\mathbf{n}}_{2}$. The outcome $s_{2}= \pm 1$ will be related to the state $P_{\hat{\mathbf{n}}_{2}}^{s_{2}}=\frac{1}{2}\left(\mathbb{I}_{2}+s_{2} \hat{\mathbf{n}}_{2} \cdot \boldsymbol{\sigma}\right)$ with probability $\operatorname{Tr}\left(P_{\mathbf{n}_{1}}^{s_{1}} P_{\hat{\mathbf{n}}_{2}}^{s_{2}}\right)=\frac{1}{2}\left(1+s_{1} s_{2} \mathbf{n}_{1} \cdot \hat{\mathbf{n}}_{2}\right)$. Hence we have

$$
\begin{aligned}
p\left(s_{1}, s_{2}\right) & =\frac{1}{2}\left(1+s_{1} \mathbf{r}_{1} \cdot \hat{\mathbf{n}}_{1}\right) \frac{1}{2}\left(1+s_{1} s_{2} \mathbf{n}_{1} \cdot \hat{\mathbf{n}}_{2}\right) \\
p\left(s_{1}\right) & =\sum_{s_{2}= \pm 1} p\left(s_{1}, s_{2}\right)=\frac{1}{2}\left(1+s_{1} \mathbf{r}_{1} \cdot \hat{\mathbf{n}}_{1}\right) \\
p\left(s_{2}\right) & =\sum_{s_{1}= \pm 1} p\left(s_{1}, s_{2}\right)=\frac{1}{2}\left[1+s_{2}\left(\mathbf{r}_{1} \cdot \hat{\mathbf{n}}_{1}\right)\left(\mathbf{n}_{1} \cdot \hat{\mathbf{n}}_{2}\right)\right]
\end{aligned}
$$

The mutual information (3) is

$$
I_{1: 2}=H_{2}\left(\frac{1+\left(\mathbf{r}_{1} \cdot \hat{\mathbf{n}}_{1}\right)\left(\mathbf{n}_{1} \cdot \hat{\mathbf{n}}_{2}\right)}{2}\right)-H_{2}\left(\frac{1+\mathbf{n}_{1} \cdot \hat{\mathbf{n}}_{2}}{2}\right),
$$

where $H_{2}(x)=-x \log _{2} x-(1-x) \log _{2}(1-x)$ is the binary entropy. The maximum of $I_{1: 2}$ is achieved for $\mathbf{r}_{1} \cdot \hat{\mathbf{n}}_{1}=0$ (i.e., the first measurement is orthogonal to the initial state, or simply the initial state is totally mixed with $\mathbf{r}_{1}=0$ ) and $\mathbf{n}_{1} \cdot \hat{\mathbf{n}}_{2}=\left|\mathbf{n}_{1}\right|$ (i.e., the second measurement is collinear to the first one). This gives the value

$$
I_{1: 2}=\log _{2} 2-H_{2}\left(\frac{1+\left|\mathbf{n}_{1}\right|}{2}\right)=1-S(\rho),
$$

which is the $d=2$ version of $S_{c}(\rho)$ as expressed in Eq. (2). Notice that the two possibilities $s_{1}= \pm 1$ of intermediate quantum state $\rho=\frac{1}{2}\left(\mathbb{I}_{2}+s_{1} \mathbf{n}_{1} \cdot \boldsymbol{\sigma}\right)$ are unitarily equivalent, so they have the same entropy $S(\rho)$. It is possible to prove the exact match between $S_{c}$ and the maximal $I_{12}$ also in arbitrary dimension [8].

Conservation of quantum information Assuming that the whole universe is in a pure state, then $\rho$ and its environment can be written in Schmidt decomposition and the entanglement entropy between them is exactly $S(\rho)$ [2, 3]. Hence, for every quantum state $\rho$ of dimension $d$ the sum of the mutual information sent in time - quantified by its coherent entropy $S_{c}(\rho)$ in Eq. $(2)$ - and the entanglement entropy $S(\rho)$ with the rest of the universe turns out to be the constant $\log _{2} d$. As a consequence, during a unitary evolution any loss of coherence is compensated by an equal increase of entanglement with the environment, and vice versa. This fact constitutes the basic statement for a conservation law of quantum information. If we interpret $S_{c}(\rho)$ as a measure of coherence of $\rho$, we obtain that Eq. 2 is an exact relation between coherence and entanglement.

Let us now consider the case of two spatially separated systems $A$ and $B$, described by an overall pure state $\rho_{A B}$. In general the state of a system is more coherent than the sum of its parts (subadditivity of entropy),

$$
S_{c}\left(\rho_{A B}\right)=S_{c}\left(\rho_{A}\right)+S_{c}\left(\rho_{B}\right)+I_{A: B}
$$

where the excess of coherent entropy amounts to the non-negative quantity $I_{A: B}=S\left(\rho_{A}\right)+S\left(\rho_{B}\right)-S\left(\rho_{A B}\right)$, which is the (spatial) mutual information between the two systems $A$ and $B$. Curiously, the coherent entropy of $\rho_{A B}$ exceeds the sum of the contributions coming from its parts $A$ and $B$ even when $I_{A: B}$ receives contribution only from classical correlations. Notice that according to Eq. (5) $S_{c}$ obeys the monotonicity property, at variance with the entropy $S$. Moreover, $S_{c}$ is a convex function in the space of density matrices.

Assuming also that $A$ and $B$ are isolated from the rest, so that unitary operations do not change the value of $S_{c}\left(\rho_{A B}\right)$, then we observe that any variation of the 
"space-like" mutual information $I_{A: B}$ is compensated by an opposite variation of the "time-like" mutual information quantified by the coherent entropy $S_{c}\left(\rho_{A}\right)+S_{c}\left(\rho_{B}\right)$.

Specifying further to the case of pure $\rho_{A B}$, we fall in the situation where $B$ is the environment of $A$, and vice versa. Now, the mutual information $I_{A: B}=2 S\left(\rho_{A}\right)=2 S\left(\rho_{B}\right)$ quantifies the entanglement between $A$ and $B$, and does not contain any contribution from classical correlations. The total quantum information consists of $S_{c}\left(\rho_{A}\right)$ bits localized in $A$, the same amount in $B$ while the remainder $I_{A: B}$ is encoded in the Hilbert space that describes both $A$ and $B$. Every unitary process will alter the balance of these quantities, without changing their sum, which is equal to the constant $\log d_{A}+\log d_{B}$, i.e. the coherent entropy of the overall pure state.

Let us assume that $A$ is localized in a well-defined region in space, delimited by a closed surface $\Sigma$. The information stored in $I_{A: B}$ can be assigned to virtual degrees of freedom assigned to the bonds connecting the real individual subsystems in $A$ and $B$. Since all these bonds cross the surface $\Sigma$, such information can be topologically located on it. In other words, during the decoherence of $A$, also $B$ decoheres, and the consequent lost information flows from both sides toward the surface: a sort of complementary of the holographic principle known in quantum gravity [9]. In this picture, a change in $I_{A: B}$ yields no net "flow of coherence" through the surface. At variance with energy, information is a scalar, so it is relativistically invariant.

In 1D lattice models, a well-known realization of such mechanism occurs when we describe matrix-product states (MPS) where the mutual information between two bipartition $A$ and $B$ of a chain is encoded in the matrices which describe the bond variables at the border between $A$ and $B$ [10].

Multi-partitions After having analyzed the case of two systems, it is interesting to understand how the quantum information carried by a quantum state of a given system is distributed when we consider its partition in several parts [11. In the case of a tripartition $A B C$, the overall coherent entropy is given by

$$
S_{c}\left(\rho_{A B C}\right)=S_{c}\left(\rho_{A}\right)+S_{c}\left(\rho_{B}\right)+S_{c}\left(\rho_{C}\right)+I_{A: B}+I_{A B: C}
$$

or cyclic permutations of subscripts $A, B, C$. The advantage of having an expression like Eq.(6), is that it involves only entropies and mutual informations, which are non negative objects quantifying amounts of information. The generalization to $n$ partitions ordered from 1 to $n$ is

$$
S_{c}\left(\rho_{1 \cdots n}\right)=\sum_{k=1}^{n} S_{c}\left(\rho_{k}\right)+I_{1: 2}+I_{12: 3}+\cdots+I_{1 \cdots(n-1): n}
$$

which can be made symmetric with respect to any label ordering.
Locally achievable coherence The simple result (2) is obtained when the optimization problem (1) is solved in the space of all the possible unitary transformations $\mathcal{U}_{\rho}$. However, one may be interested to restrict the calculation to the family of local transformations with respect of a given partition. For a bipartite state $\rho_{A B}$ we can define

$$
S_{c}^{l o c}\left(\rho_{A B}\right)=\max _{\sigma \in \mathcal{U}_{\rho_{A B}}^{\text {loc }}}[\tilde{S}(\sigma)]-\min _{\sigma \in \mathcal{U}_{\rho_{A B}^{l o c}}^{\text {loc }}}[\tilde{S}(\sigma)],
$$

where where $\mathcal{U}_{\rho_{A B}}^{\text {loc }}$ is the set of all matrices which are equivalent to $\rho_{A B}$ under local unitaries $U_{A} \otimes U_{B}$. The result of such an optimization is not guaranteed to give the same clean expression as in Eq. (2); instead we expect a lesser value which must be calculated numerically. It is appropriate to define the coherence gap $G\left(\rho_{A B}\right)=S_{c}\left(\rho_{A B}\right)-S_{c}^{l o c}\left(\rho_{A B}\right)$, namely the information which cannot be accessed by local operations. The quantity $G\left(\rho_{A B}\right)$ accounts for nonlocal correlations between $A$ and $B$ (not necessarily the entanglement) in a similar fashion as the quantum discord [12, or the deficit [7]. The remaining local correlations between $A$ and $B$ are quantified by $L\left(\rho_{A B}\right)=I_{A: B}-G\left(\rho_{A B}\right)$.

Examples In order to familiarize with the concepts discussed in this paper, we analyze the repartition of information in some quantum states.

- The Bell state $\left|\Psi^{+}\right\rangle=(|00\rangle+|11\rangle) / \sqrt{2}$ is a pure state with $d=4$, i.e. $S_{c}\left(\rho_{A B}\right)=2$, meaning 2 bits of information. After partial trace we get $\rho_{A}=\operatorname{Tr}_{B}\left(\left|\Psi^{+}\right\rangle\left\langle\Psi^{+}\right|\right)=\frac{1}{2}(|0\rangle\langle 0|+| 1\rangle\langle 1|)$, so both the subsystems are totally mixed, with $S_{c}\left(\rho_{A}\right)=$ $S_{c}\left(\rho_{B}\right)=0$. The two bits are stored in the mutual information is $I_{A: B}=2$, which we can figure out as localized on the whole system $A B$, while $A$ and $B$ are separately incoherent. Notice that one bit is due to entanglement entropy, $S\left(\rho_{A}\right)=1$, while the remaining bit involves the classical parity correlations [3]. Remarkably, $G\left(\rho_{A B}\right)=1$ is the same as the entanglement entropy.

- The three-site GHZ state $\left|\Psi_{G H Z}\right\rangle=(|000\rangle+$ $|111\rangle) / \sqrt{2}$, a paradigmatic example where tripartite entanglement is present, while the pairwise one is zero. The single quantities are summarized in the following table:

\begin{tabular}{|c|c|c|c|c|c|c|c|}
\hline$\left|\Psi_{G H Z}\right\rangle$ & $\rho_{A}$ & $\rho_{B}$ & $\rho_{C}$ & $\rho_{A B}$ & $\rho_{A C}$ & $\rho_{B C}$ & $\rho_{A B C}$ \\
\hline \hline$S$ & 1 & 1 & 1 & 1 & 1 & 1 & 0 \\
\hline$S_{c}$ & 0 & 0 & 0 & 1 & 1 & 1 & 3 \\
\hline$G$ & & & & 0 & 0 & 0 & 1 \\
\hline$L$ & & & & 1 & 1 & 1 & \\
\hline$I$ & & & & 1 & 1 & 1 & \\
\hline$E_{f}$ & & & & 0 & 0 & 0 & \\
\hline
\end{tabular}

where we have also included a row for the entanglement of formation $E_{f}$, which is exactly computable 
for pairs of qubits [13]. The single subsystems $A$, $B$ and $C$ are all totally incoherent. Three qubits are stored in the mutual information between pairs, all made of local correlations. Both the nonlocal indicators $G$ and $E_{f}$ are vanishing between pairs. Interestingly, $G$ can be computed also for the tripartite case, resulting in one nonlocally achievable bit.

- The three-site W state $\left|\Psi_{W}\right\rangle=(|001\rangle+|010\rangle+$ $|100\rangle) / \sqrt{3}$. In this case, we have

\begin{tabular}{|c|c|c|c|c|c|c|c|}
\hline$\left|\Psi_{W}\right\rangle$ & $\rho_{A}$ & $\rho_{B}$ & $\rho_{C}$ & $\rho_{A B}$ & $\rho_{A C}$ & $\rho_{B C}$ & $\rho_{A B C}$ \\
\hline \hline$S$ & 0.918 & 0.918 & 0.918 & 0.918 & 0.918 & 0.918 & 0 \\
\hline$S_{c}$ & 0.082 & 0.082 & 0.082 & 1.082 & 1.082 & 1.082 & 3 \\
\hline$G$ & & & & 0.667 & 0.667 & 0.667 & 1.667 \\
\hline$L$ & & & & 0.252 & 0.252 & 0.252 & \\
\hline$I$ & & & & 0.918 & 0.918 & 0.918 & \\
\hline$E_{f}$ & & & & 0.550 & 0.550 & 0.550 & \\
\hline
\end{tabular}

Now, some information is carried by single sites, while 0.918 bits is stored in pairwise correlations: 0.252 local and 0.667 nonlocal. The presence of nonlocal correlations is confirmed also by 0.550 bits of entanglement of formation.

Conclusions This paper illustrates some arguments which lead to the definition of an entropic value coming from coherent information in quantum states. Such a quantity, here called coherent entropy, is indeed physical as it quantifies the (mutual) information conveyed in time by quantum states; so it is necessary in order to give a complete description of their informational content. By means of this quantity and ordinary mutual information between different systems, it is possible to write equations of conservation of information in multipartite states, during unitary processes. Looking at a specific part of an interacting system, we observe that "time-like" information is transformed into "space-like" one: the overall information is conserved and the "flow" through a closed surface is governed by a holographic principle.

As the universe is believed to obey to quantum mechanics where time evolution is unitary (dissipation and decay processes are not, because they are only partial descriptions) it is imperative to elevate conservation of information to a fundamental concept and taking advantage of it, like it happens with any other conserved quantity. A remarkable consequence is that no information has been generated or lost since creation of the universe, but it has only spread out due to expansion and interactions. The space-time symmetric treatment of mutual information suggests a possible use in general relativity. For instance, it could help to shed some light in solving the famous paradox of information loss in black holes 9, 14. The change of metric signature after crossing the event horizon could be responsible of the transformation of space-like information into time-like, i.e. a purification of quantum states. This is notoriously connected with the interpretation of the measurement postulate in quantum mechanics which invokes a collapse of the wavefunction after extracting some information about the original state. On the contrary, in the present framework the consequence of a projective measurement is to inject quantum (coherent) information into a state, as the output quantum state is pure.

Finally, it would be interesting to explore other possible consequences of conservation of coherent information in foundations of quantum mechanics. We believe that the vision described in the present work could yield some interesting implications also in field theories and statistical mechanics.

Acknowledgements Many thanks to Lorenzo Campos Venuti per very helpful discussions. This paper is dedicated to the memory of my friend Roberto Ghedini, who was the most sincere person I ever knew.

* marco.roncaglia.it@gmail.com

[1] M. Horodecki, R. Horodecki, A. Sen(De), and U. Sen, Found. Phys. 35, 2041 (2005).

[2] M. A. Nielsen and I. L. Chuang, Quantum Computation and Quantum Information (Cambridge University Press, 2000).

[3] J. Preskill, Quantum Information and Computation (Lecture Notes for Physics 229, California Institute of Technology, 1998).

[4] J. Åberg, arXiv:quant-ph/0612146, T. Baumgratz, M. Cramer, and M. B. Plenio, Phys. Rev. Lett. 113, 140401 (2014).

[5] A. Wehrl, Rev. Mod. Phys. 50, 221 (1978).

[6] J. Oppenheim, M. Horodecki, P. Horodecki, and R. Horodecki, Phys. Rev. Lett. 89, 180402 (2002).

[7] M. Horodecki, K. Horodecki, P. Horodecki, R. Horodecki, J. Oppenheim, A. Sen, and U. Sen, Phys. Rev. Lett. 90, 100402 (2003).

[8] The proof is illustrated in the Supplemental Material.

[9] L. Susskind, J. Math. Phys. 36, 6377 (1995).

[10] M. Fannes, B. Nachtergaele and R.F. Werner, Commun. Math. Phys. 144, 443 (1992).

[11] A.C.S. Costa, R.M. Angelo, and M.W. Beims, Phys. Rev. A 90, 012322 (2014).

[12] H. Ollivier and W.H. Zurek, Phys. Rev. Lett. 88, 017901 (2001).

[13] W. K. Wootters, Phys. Rev. Lett. 80, 2245 (1998).

[14] S. L. Braunstein and A. K. Pati, Phys. Rev. Lett. 98, 080502 (2007). 


\section{SUPPLEMENTAL MATERIAL}

\section{Maximal mutual information in time for states in arbitrary dimension}

Referring to the scheme in Fig 2 , the maximum of $I_{1: 2}$ must be calculated with respect to every measurement at $t=t_{1}$ and $t=t_{2}$ of the complete observable $A$ and $B$, respectively. The first measurement $A$ on the initial state $d$-dimensional $\rho_{\text {in }}$ will generate an output signal $s_{1}=1, \ldots, d$, producing a new state equal to the projector $P_{A}^{s_{1}}=\left|s_{1}\right\rangle_{A}\left\langle\left. s_{1}\right|_{A}\right.$, with probability $\operatorname{Tr}\left(\rho_{\text {in }} P_{A}^{s_{1}}\right)$. Successively, the system will be subjected to some decoherence, which, in general, can be modeled in the operator sum formalism as

$$
\rho=\sum_{m} M_{m} P_{A}^{s_{1}} M_{m}^{\dagger}
$$

with Kraus operators obeying the completeness relation $\sum_{m} M_{m}^{\dagger} M_{m}=\mathbb{I}$. It is important to consider that in general the intermediate state $\rho$ depends on the first outcome $s_{1}$. For our scopes, all these intermediate states should be unitarily equivalent, in order to correctly quantify the information carried by the given state $\rho$. Below, we show that such situation is always made possible by a choice for the $M_{m}$ 's. Finally, at time $t_{2}$ the quantum state undergoes another measurement $B$, generating the output $s_{2}$, with outcoming state $P_{A}^{s_{2}}$, with probability $\operatorname{Tr}\left(\rho P_{A}^{s_{2}}\right)$.

In this process, the joint and marginal probability distributions are given by

$$
\begin{aligned}
p\left(s_{1}, s_{2}\right) & =\operatorname{Tr}\left(\rho_{\text {in }} P_{A}^{s_{1}}\right) \operatorname{Tr}\left(\rho_{s_{1}} P_{B}^{s_{2}}\right) \\
p\left(s_{1}\right) & =\operatorname{Tr}\left(\rho_{\text {in }} P_{A}^{s_{1}}\right) \sum_{s_{2}} \operatorname{Tr}\left(\rho_{s_{1}} P_{B}^{s_{2}}\right)=\operatorname{Tr}\left(\rho_{\text {in }} P_{A}^{s_{1}}\right) \\
p\left(s_{2}\right) & =\sum_{s_{1}} \operatorname{Tr}\left(\rho_{\text {in }} P_{A}^{s_{1}}\right) \operatorname{Tr}\left(\rho_{s_{1}} P_{B}^{s_{2}}\right)
\end{aligned}
$$

where we have obviously used the completeness relation $\sum_{s} P_{\alpha}^{s}=\mathbb{I}, \alpha=A, B$. Moreover we have specified the index in the intermediate state $\rho_{s_{1}}$, remembering that they have the same eigenvectors and eigenvalues, possibly in different orders.

So, the mutual information can be written as

$$
I_{1: 2}=\sum_{s_{1}, s_{2}} p\left(s_{1}, s_{2}\right) \log _{2}\left(\frac{p\left(s_{1}, s_{2}\right)}{p\left(s_{1}\right) p\left(s_{2}\right)}\right)=C_{1}-C_{2}
$$

with

$$
\begin{aligned}
& C_{1}=-\sum_{s_{2}} p\left(s_{2}\right) \log _{2} p\left(s_{2}\right) \\
& C_{2}=-\sum_{s_{1}} \operatorname{Tr}\left(\rho_{\text {in }} P_{A}^{s_{1}}\right) \sum_{s_{2}} \operatorname{Tr}\left(\rho_{s_{1}} P_{B}^{s_{2}}\right) \log _{2}\left(\operatorname{Tr}\left(\rho_{s_{1}} P_{B}^{s_{2}}\right)\right)
\end{aligned}
$$

where $C_{1}$ is the Shannon entropy of the second measurement $H\left(\left\{s_{2}\right\}\right)$, and $C_{2}$ is the conditional entropy
$H\left(\left\{s_{2}\right\} \mid\left\{s_{1}\right\}\right)$. These two terms can be optimized separately in order to find the maximum value of $I_{1: 2}$ in the space of all the measurement basis $A$ and $B$. Let us observe that $\operatorname{Tr}\left(\rho_{s_{1}} P_{B}^{s_{2}}\right)$ are just the diagonal elements $\left(s_{2}=1, \ldots, d\right)$ of $\rho_{s_{1}}$ in the $B$ basis. If we indicate with $\tilde{\rho}_{s_{1}}$ the diagonal part of $\rho_{s_{1}}$ in the $B$ basis, we obtain

$$
C_{2}=\sum_{s_{1}} \operatorname{Tr}\left(\rho_{\mathrm{in}} P_{A}^{s_{1}}\right) S\left(\tilde{\rho}_{s_{1}}\right)
$$

which is clearly minimal when $B$ is the basis of eigenvectors of all the $\rho_{s_{1}}$ 's, giving $C_{2}=S(\rho)$, because all the $\rho_{s_{1}}$ 's have the same eigenvalues. Denoting with $\lambda_{i}$ $(i=1, \ldots, d)$ the eigenvalues of $\rho_{1}$, it is easy to see that the term $C_{1}$ reaches its theoretical maximum $\log _{2} d$, with the choice

$$
\left\{\begin{array}{l}
\operatorname{Tr}\left(\rho_{s_{1}} P_{B}^{s_{2}}\right)=\lambda_{\left(s_{1}+s_{2}-1\right) \bmod d}, \\
\operatorname{Tr}\left(\rho_{\text {in }} P_{A}^{s_{1}}\right)=1 / d,
\end{array} \forall s_{1}=1, \ldots, d\right.
$$

In other words, the spectra of the density matrices $\rho_{s_{1}}$ are given by all the cyclic permutations of $\left\{\lambda_{1}, \lambda_{2}, \ldots, \lambda_{d}\right\}$. In this way, we get $\sum_{s_{1}} \operatorname{Tr}\left(\rho_{s_{1}} P_{B}^{s_{2}}\right)=1, \forall s_{2}$. The explicit form of the Kraus operators for obtaining the first row of Eq. (8) is

$$
M_{m}=\sum_{s=1}^{d} P_{B}^{m} \Pi_{B}^{s-1}\left(\Pi_{A}^{s-1}\right)^{\dagger} P_{A}^{s}
$$

with the condition $\operatorname{Tr}\left(P_{A}^{1} P_{B}^{m}\right)=\lambda_{m}$, which determines what the basis $A$ should be. The $n$-steps cyclicpermutation operator of vectors $\left\{|i\rangle_{A}\right\}_{i=1}^{d}$ in a given $d$ dimensional orthogonal basis $A$ is

$$
\Pi_{A}^{n}=\sum_{k}|k+n\rangle_{A}\left\langle\left. k\right|_{A}\right.
$$

Moreover, in the second row of (8) we have required that $\rho_{\text {in }}$ at $t_{1}$ gives all the possible outcomes with equal probability.

In summary, we have obtained that the maximal amount of information conveyed between past and future measurements is

$$
I_{1: 2}=\log _{2} d-S(\rho)
$$

namely equal to the coherent entropy of $\rho$ expressed in Eq. (2).

\section{Every quantum state admit a measurement with completely random outputs}

The statement can be reformulated through the following theorem. 
Theorem. Let $\rho$ be a $d$-dimensional square matrix with spectrum $\left\{r_{j}, j=1, \ldots, d\right\}$ and related orthonormalized eigenvectors $\left|r_{j}\right\rangle$. Then, it exists a basis of orthonormal vectors $\left\{\left|\phi_{k}\right\rangle, k=1, \ldots, d\right\}$ where the all the diagonal elements of $\rho$ are equal.

Proof - We can proceed in a constructive way by writing down the explicit transformation

$$
\left|\phi_{k}\right\rangle=\frac{1}{\sqrt{d}} \sum_{j=1}^{d} \exp \left(\frac{2 \pi i j k}{d}\right)\left|r_{j}\right\rangle .
$$

Let us check that the $\left|\phi_{k}\right\rangle$ are orthonormal

$$
\begin{aligned}
\left\langle\phi_{k^{\prime}} \mid \phi_{k}\right\rangle & =\frac{1}{d} \sum_{j, j^{\prime}=1}^{d} \exp \left(-\frac{2 \pi i j^{\prime} k^{\prime}}{d}\right) \exp \left(\frac{2 \pi i j k}{d}\right)\left\langle r_{j^{\prime}} \mid r_{j}\right\rangle \\
& =\frac{1}{d} \sum_{j=0}^{d-1} \exp \left[\frac{2 \pi i\left(k-k^{\prime}\right)}{d} j\right]=\delta_{k k^{\prime}}
\end{aligned}
$$

which assures the unitarity of the transformation in Eq. (9). The diagonal terms are

$$
\begin{aligned}
\left\langle\phi_{k}|\rho| \phi_{k}\right\rangle & =\frac{1}{d} \sum_{j, j^{\prime}=1}^{d} \exp \left(-\frac{2 \pi i j^{\prime} k}{d}\right) \exp \left(\frac{2 \pi i j k}{d}\right)\left\langle r_{j^{\prime}}|\rho| r_{j}\right\rangle \\
& =\frac{1}{d} \sum_{j=1}^{d} r_{j}=\frac{1}{d} \operatorname{Tr}(\rho)
\end{aligned}
$$

for every $k$.

In particular, if $\rho$ is a density matrix, we have $\operatorname{Tr}(\rho)=$ 1 , so all the diagonal elements in the basis $\left|\phi_{k}\right\rangle$ are all equal to $1 / d$. Notice that the choice 9 is not unique, because we have the freedom to perform the gauge transformation $\left|r_{j}\right\rangle \rightarrow \exp \left(i \theta_{j}\right)\left|r_{j}\right\rangle$.

The result of the present theorem was discussed as a guided exercise (Problem 3, Sect. 2.2) in the book by R. A. Horn and C. R. Johnson, Matrix Analysis, (Cambridge University Press, 1985) to be solved through an iterated inverse Jacobi procedure that maximizes the 2-norm of the off-diagonal part $\sum_{i \neq j}\left|\rho_{i j}\right|^{2}$. 